\title{
LSm1 binds to the Dengue virus RNA 3' UTR and is a positive regulator of Dengue virus replication
}

\author{
YANGCHAO DONG $^{1 *}$, JING YANG $^{1 *}$, WEI YE ${ }^{1 *}$, YUAN WANG $^{1}$, YUNBO MIAO $^{1}$, \\ TIANBING DING $^{1}$, CHEN XIANG $^{2}$, YINGFENG LEI ${ }^{1}$ and ZHIKAI XU ${ }^{1}$ \\ ${ }^{1}$ Department of Microbiology, Faculty of Preclinical Medicine, \\ The Fourth Military Medical University, Xi'an, Shaanxi 710032; ${ }^{2}$ Department of Orthopaedics, \\ Tangdu Hospital, The Fourth Military Medical University, Xi'an, Shaanxi 710038, P.R. China
}

Received June 13, 2014; Accepted February 25, 2015

DOI: 10.3892/ijmm.2015.2169

\begin{abstract}
Dengue virus (DENV) is a mosquito-transmitted flavivirus that can cause severe disease in humans. The DENV positive strand RNA genome contains $5^{\prime}$ and $3^{\prime}$ untranslated regions (UTRs) that have been shown to be required for virus replication and interaction with host cell proteins. In the present study LSm1 was identified as a host cellular protein involved in DENV RNA replication. By using two independent methodologies, we demonstrated a critical interaction between LSm1 and the 3' UTR of DENV. Furthermore, the confocal immunofluorescence analysis showed that the interaction between LSm1 and viral RNA is located in P-body around nucleoli in the cytoplasm. LSm1 knockdown by siRNA specifically reduced the levels of viral RNA in DENV-infected cells and infectious DENV particles in the supernatant. These results provide evidence that LSm1 binding to the DENV RNA 3' UTR positively regulates DENV RNA replication.
\end{abstract}

\section{Introduction}

The four Dengue viruses (DENV 1-4) are enveloped, positive strand RNA viruses, and cause a broad spectrum of clinical manifestations (1). The DENV, as well as West Nile virus (WNV), Japanese encephalitis virus (JEV) and Hepatitis C virus (HCV) belongs to the family Flaviviridae, which follow the similar replication mechanisms (2). The DENV RNA is translated into a single poly-protein precursor that is subsequently cleaved into

Correspondence to: Professor Zhikai Xu or Dr Yingfeng Lei, Department of Microbiology, Faculty of Preclinical Medicine, The Fourth Military Medical University, 169th Changle West Road, Xi'an, Shaanxi 710032, P.R. China

E-mail: zhikaixu523@163.com

E-mail: yflei@fmmu.edu.cn

*Contributed equally

Key words: Dengue virus, LSm1, host protein, processing body, viral replication three structural proteins and seven non-structural proteins. The DENV NS3 and NS5 are directly involved in viral RNA replication, with NS3 acting as an RNA helicase and NS5 acting as the RNA-dependent RNA polymerase (2). In addition, DENV is dependent on host factors to establish successful infection and accomplish viral replication (2).

Several RNA binding proteins have been reported to be involved in the replication of DENV RNA. YocupicioMonroy et al $(3,4)$ reported that the La, calreticulin, and protein disulfide isomerase proteins from a human monocytic cell line bind to the $3^{\prime}$ untranslated region (UTR) of DENV-4, and La protein interacts with the viral 3' UTRs and is redistributed in DENV-infected cells. Another study by De Nova-Ocampo et al (5) showed that the La protein, translation elongation factor- $1 \alpha(E F-1 \alpha)$, and polypyrimidine tract-binding protein (PTB) interact with the genomic 3' UTR of DENV-4 in which the CS1-L-SL region is responsible for forming specific and stable complexes with cellular proteins. Additionally, different regions located in the DENV 3' UTR (+) have been shown to interact with several other proteins such as poly(A)-binding protein, NF90, p100, Y box-binding protein-1, HNRNPs A1, A2/B2 and Q (6-9). Ward et al (10) reported that one of the P-body protein DDX6 bound to DENV RNA during infection and this interaction was mediated by the DB1 and DB2 structures in the 3' UTR, whereas the stress granule (SG) proteins G3BP1, Caprin1 and USP10 bound to the variable region (VR) in the 3' UTR. Considering that those proteins colocalized with DENV replication sites and bound to different sites on 3' UTR, they suggested that the DENV-2 RNA was guided to a site for assembly of P-body and SG proteins which could regulate whether the viral RNA undergoes translation, replication or packaging. Further identification of host factors interacting with DENV RNA may improve our understanding of the DENV amplification cycle.

The LSm1-7 complex is known to be an important component of the P-body, and acts as the highly conserved RNA-binding protein to regulate the fate of cellular mRNAs $(11,12)$. The LSm1-7 complex is also involved in the replication of several viruses. A screen for host factors required for Brome mosaic virus (BMV) gene expression identified that LSm1-7 complex plays an essential role in translation and in the translation-replication transit of the BMV genome $(13,14)$. 
Pérez-Vilaró et al (15) and Scheller et al (16) showed that the LSm1-7 complex is required for efficient translation of the $\mathrm{HCV}$ genome and that these requirements are functionally linked to the 5' and 3' UTRs, which are known to play key roles in the regulation of HCV translation and replication.

Given the conserved function of the LSm1-7 complex in the mRNA processing and the common mechanism of all (+) RNA viruses replication, we hypothesized that the LSm1-7 complex may be used by DENV RNA to replicate in human cells. In the present study, we showed that LSm1 can interact with DENV RNA specifically. By measuring DENV RNA amplification and infectious virus particle production, our results indicated that the LSm1-7 complex is necessary for DENV replication.

\section{Materials and methods}

Cell culture and cytoplasmic extract. A549 and Vero-E6 cells were maintained in RPMI-1640 (Invitrogen, Carlsbad, CA, USA) supplemented with 10\% FBS (Hyclone Laboratories Inc., Logan, UT, USA), $10 \mathrm{U} / \mathrm{ml}$ penicillin and $10 \mu \mathrm{g} / \mathrm{ml}$ streptomycin (Pen/Strep; Gibco-Invitrogen, Carlsbad, CA, USA) at $37^{\circ} \mathrm{C}$ in a $5 \% \mathrm{CO}_{2}$ atmosphere. $\mathrm{C} 6 / 36$ (Aedes albopictus) cells were cultured at $28^{\circ} \mathrm{C}$ in RPMI-1640 supplemented with $10 \%$ FBS.

For preparation of the cytoplasmic extract (S-100), A549 cells were adapted for growth on a large scale. Cell extracts from A549 cells were prepared using the procedure as described by Paranjape and Harris (9).

DENV-2 amplification and infection. DENV-2 was amplified in C6/36 cells as previously described (17). For DENV-2 infection, the cells were incubated with the virus (MOI=1) for $2 \mathrm{~h}$ at $37^{\circ} \mathrm{C}$ with occasional rocking. After $2 \mathrm{~h}$, the cells were rinsed, overlaid with complete medium, and incubated at various time points.

Immunofluorescence assay and confocal microscopy. A549 cells were seeded onto 8-well coverslips (LabTek, Bloomington, IN, USA) and infected with DENV-2 for $24 \mathrm{~h}$. After being fixed and permeabilized, the cells were incubated with blocking buffer [PBS containing 3\% bovine serum albumin (BSA)] for $1 \mathrm{~h}$. Primary antibodies were diluted in blocking buffer $(1: 300$ for anti-LSm1 and anti-Dcp1B, Proteintech Group, Chicago, IL, USA; 1:200 for anti-dsRNA, English and Scientific Consulting Kft., Szirák, Hungary) and incubated with cells at $4^{\circ} \mathrm{C}$ overnight. The cells were then washed and incubated for $1 \mathrm{~h}$ with fluorescently labelled Alexa Fluor 488 goat anti-rabbit and Alexa Fluor 594 goat anti-mouse antibodies (Invitrogen). Coverslips were mounted in ProLong Gold antifade reagent containing DAPI (Invitrogen), and the cells were visualized under a laser confocal microscope LSM 510 (Carl Zeiss, Inc., Thornwood, NY, USA). The images were captured and analyzed by using ZEN software (Carl Zeiss, Inc.).

RNA interference. The siRNAs, target LSm1 (siLSm1: 5'-CAA ACUUAGUGCUACAUCATT-3') and the scrambled sequence as a negative control (siNC: 5'-UUCUCCGAACGUGUCACG UTT-3') were transfected into A549 cells with 33 and $25 \mathrm{nM}$ of each siRNA for the first and second transient transfections, respectively. The effects of LSm1 knockdown were detected
$48 \mathrm{~h}$ post-secondary transfection by immunoblotting LSm1 protein and mRNA levels. For detection of the effect of LSm1 knockdown on DENV replication, siRNA-transfected A549 cells were infected with DENV-2. At the indicated time points, the supernatant and cell RNAs were collected for infection of Vero-E6 cells and relative reverse transcription quantitative polymerase chain reaction (RT-qPCR) analysis, respectively.

Relative RT-qPCR analysis. LSm1 gene expression and viral RNA synthesis were analyzed by relative quantitative RT-PCR (primers: LSm1-F, 5'-GACTTGGAAAAGGAG AGTGACA-3'; LSm1-R, 5'-GGGCAAAAGATTAGTACT CATC-3'; DENV-NS5-F, 5'-ACAAGTAGAACAACCTGG TCCAT-3'; DENV-NS5-R: 5'-GCCGCACCATTGGTCTT CTC-3'). The housekeeping gene $\beta$-actin ( $\beta$-actin-F, 5'-TGA CGGGGTCACCCACACT G-3'; $\beta$-actin-R, 5'-AAGCTG TAGCCGCGCTCGGT-3') was used as an internal control. Total RNA was isolated by using RNAiso and total cDNA was synthesized by using PrimeScript ${ }^{\mathrm{TM}}$ RT Master Mix (both from Takara Bio, Inc., Shiga, Japan). Each cDNA was amplified with the above primers and SYBR Premix Ex Taq ${ }^{\mathrm{TM}}$ II (Takara Bio, Inc.) for 40 cycles and data were analyzed by using Mx3005P System Software (Stratagene, La Jolla, CA, USA). Relative gene expression refers to the magnitude of the signal generated for the NS5 gene of DENV from siLSm1- and siNC-treated cells using the $\beta$-actin gene as the internal control.

Western blotting. The samples were separated by SDS-PAGE followed by immunoblotting analysis using a rabbit polyclonal anti-LSm1 or a mouse anti- $\beta$-actin mAb (both from Proteintech Group).

RNA immunoprecipitation. The interaction of LSm1 with DENV viral RNA during infection was studied by using RNA immunoprecipitation assay. Briefly, the infected cells were collected and cross-linked with $0.1 \%$ formaldehyde. The cells were then disrupted by sonication in RIP assay buffer $(50 \mathrm{mM}$ Tris- $\mathrm{HCl}, \mathrm{pH} 7.5,1 \%$ Nonidet $\mathrm{P}-40,0.5 \%$ sodium deoxycholate, $0.05 \%$ SDS, $1 \mathrm{mM}$ EDTA and $150 \mathrm{mM} \mathrm{NaCl}, 0.2 \mathrm{mM}$ PMSF, $1 \mathrm{X}$ protease inhibitors and 40 units RNase inhibitor), and the resulting lysates were precleared with protein A-agarose (Santa Cruz Biotechnology, Inc., Santa Cruz, CA, USA). Immunoprecipitation was carried out using anti-LSm1 monoclonal antibody or normal rabbit $\operatorname{IgG}$ (as a negative control). Immune complexes were precipitated with protein A-agarose. Viral RNA immunoprecipitation was isolated using an RNeasy mini kit (Qiagen). RT-PCR was conducted using primers $3^{\prime}$ UTR-F (5'-AAGGCAAAACTAACATGA AAC-3') and 3' UTR-R (5'-AGAACCTGTTGATTCAAC AGC-3').

RNA pull-down assay. To create DNA templates for the in vitro transcription of DENV 3' UTR, plasmid FL-D2 (18) containing the full-length DENV-2 genome was used as the template to amplify 454 nt of the DENV 3' UTR. PCR was performed with primers $3^{\prime}$ UTR-F (5'-TAATACGACTCACT ATAGGGAAGGCAAAACTAACATGAAAC-3'), including the T7 promoter and 3' UTR-R. PCR templates were used for T7 RiboMAX Express Large Scale RNA Production 
$\mathbf{A}$

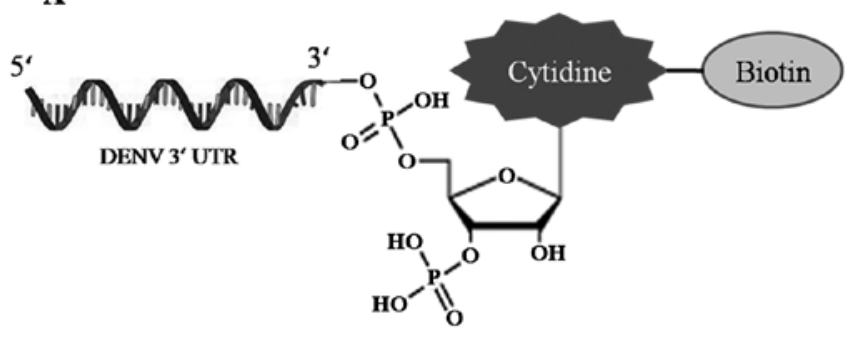

Biotin labeled DENV $3^{\prime}$ UTR

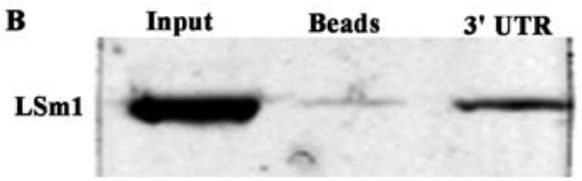

Figure 1. Dengue virus (DENV) RNA 3' untranslated region (UTR) interacts with LSm1 in vitro. (A) Schematic representation of biotin-labeled 3' UTR used in the RNA pull-down assay. (B) Paramagnetic streptavidin beads bound with biotinylated 3' UTR transcript were incubated with cell cytoplasmic extracts S-100 in the presence of a 10-fold excess of yeast tRNA to inhibit non-specific binding. The bound RNA-protein complexes were pulled down with streptavidin beads, analyzed and separated using western blotting.

System (Promega, Madison, WI, USA) to generate DENV 3' UTR RNA according to the manufacturer's instructions. To generate DENV RNA 3' UTR-Bio, the Pierce ${ }^{\mathrm{TM}}$ RNA 3 End Biotinylation kit (Thermo Scientific, Waltham, MA USA) was used to attach a single biotinylated nucleotide to the $3^{\prime}$ terminus of a 3' UTR RNA strand.

The pull-down assay was then performed. Briefly, $25 \mu \mathrm{g}$ of DENV RNA 3' UTR-Bio in RNA binding buffer $(150 \mathrm{mM}$ $\mathrm{NaCl}, 50 \mathrm{mM}$ Hepes-HCl pH 7.5, 0.5\% NP-40) was heated to $95^{\circ} \mathrm{C}$ for $5 \mathrm{~min}$, cooled to room temperature, combined with paramagnetic streptavidin beads (Dynabeads C1, Dynabeads ${ }^{\circledR}$ MyOne $^{\text {TM }}$ Streptavidin C1, Invitrogen) and incubated on a rotation wheel at $4^{\circ} \mathrm{C}$ for $30 \mathrm{~min}$. The beads were then washed three times with RNA washing buffer $(250 \mathrm{mM} \mathrm{NaCl}$, $50 \mathrm{mM}$ Hepes-HCl pH 7.5, 0.5\% NP-40 and $10 \mathrm{mM} \mathrm{MgCl} 2$ ) prior to incubation with $400 \mu \mathrm{g}$ of S-100 fraction containing 40 units RNase inhibitor (Takara Bio, Inc.) and $20 \mu \mathrm{g}$ yeast tRNA (Invitrogen) by agitation for $30 \mathrm{~min}$ at $4^{\circ} \mathrm{C}$. After incubation beads were washed another three times with RNA wash buffer, fractions from the beads were boiled for $5 \mathrm{~min}$ in $0.1 \%$ SDS for subsequent western blotting.

Flow cytometric analysis. Vero-E6 cells were infected with DENV-2 containing supernatant from LSm1 knockdown cells or siNC-treated cells infected with DENV-2 in 6-well culture plates. Thirty-six hours post-infection, the cells were collected and treated in $100 \mu \mathrm{l}$ of Cytofix/Cytoperm buffer (Becton-Dickinson) and then washed according to the manufacturer's instructions. The cells were resuspended with the DENV antibody 4G2 conjugated to fluorescein isothiocyanate (FITC) (A190-108F; Cambridge Bioscience) and incubated for $1 \mathrm{~h}$ at $4{ }^{\circ} \mathrm{C}$, followed by two rounds of washing, and then resuspended in $300 \mu 1$ of PBS. Flow cytometry was carried out with a BD LSR II instrument, with data analysis using FlowJo software (Tree Star, Inc., Ashland, OR, USA).

\section{Results}

The host factor LSm1 interacts with DENV RNA in vitro. LSm1 has been shown to be involved in the propagation of the positive strand RNA virus BMV and HCV. Following infection with these viruses, LSm1 interacts directly with viral RNA genomes via the 5' and 3' UTRs. To investigate whether LSm1 was present in the ribonucleoprotein (RNP) complex formed between the DENV-2 RNA and host factors, an RNA pull-down assay was performed. The paramagnetic streptavidin beads-based RNA pull-down assay using biotinylated DENV RNA 3' UTR (Fig. 1A) was performed. Purified DENV 3' UTR-Bio RNA was bound to beads and incubated with A549 cell S-100 fractions, and the interacting proteins were analyzed by western blotting. DENV 3' UTR-Bio RNA but not beads alone was able to precipitate LSm1 present in cell extracts (Fig. 1B, left). As indicated from this result LSm1 can interact with DENV RNA specifically in vitro.

LSm1 associates with DENV RNA in infected cells and colocalizes with the DENV replication complex. To determine whether LSm1 associates with DENV RNA during a virus infection, a viral RNA immunoprecipitation assay was performed using anti-LSm1 antibody. A549 cells were infected with DENV-2 at an MOI of 1. Cells were harvested $24 \mathrm{~h}$ post-inoculation (p.i.) and treated with $0.1 \%$ formaldehyde to cross-link protein RNA complexes. After the cell lysates were incubated with anti-LSm1 antibody, the RNA-LSm1-7 complexes were isolated after high stringency washing and RNA was extracted. RT-PCR was conducted with the primers targeted to DENV 3' UTR. As shown in Fig. 2A, DENV RNA bound to LSm1 was detected 24 h p.i. Normal rabbit IgG precipitation controls run in parallel were negative. These results suggested that LSm1 interacts with DENV RNA during the course of DENV-2 infection in vivo.

To determine the potential role of LSm1 in the DENV life cycle, we examined the subcellular localization of LSm1 during DENV-2 infection using confocal laser scanning microscopy. To detect DENV RNA, we used an antibody specific to dsRNA that was used previously as a marker for sites of DENV replication (19). A549 cells were infected with DENV-2 at an MOI of 1. After $24 \mathrm{~h}$, the cells were fixed, permeabilized and stained with anti-dsRNA and anti-LSm1 antibody. For comparison, uninfected cells were stained with the same antibodies. Nuclei were stained with DAPI, and the coverslips were analyzed under a confocal laser scanning microscope. In the uninfected cells, we observed detectable LSm1 exclusively. In the infected cells, LSm1 localized to sites of DENV replication were indicated by dsRNA signal (Fig. 2B).

LSml depletion inhibits the replication of DENV RNA and release of infectious particles. LSm1 has been previously involved in HCV replication and translational silencing. To determine whether LSm1 has a potential role in DENV-2 replication, we established silencing conditions for LSm1 in A549 cells followed by DENV-2 infection and analyzed the levels of viral RNA in the infected cells and infectious viral particles in the supernatant. The results showed that targeting LSm1 siRNA-mediated silencing specifically reduced $71.71 \%$ 

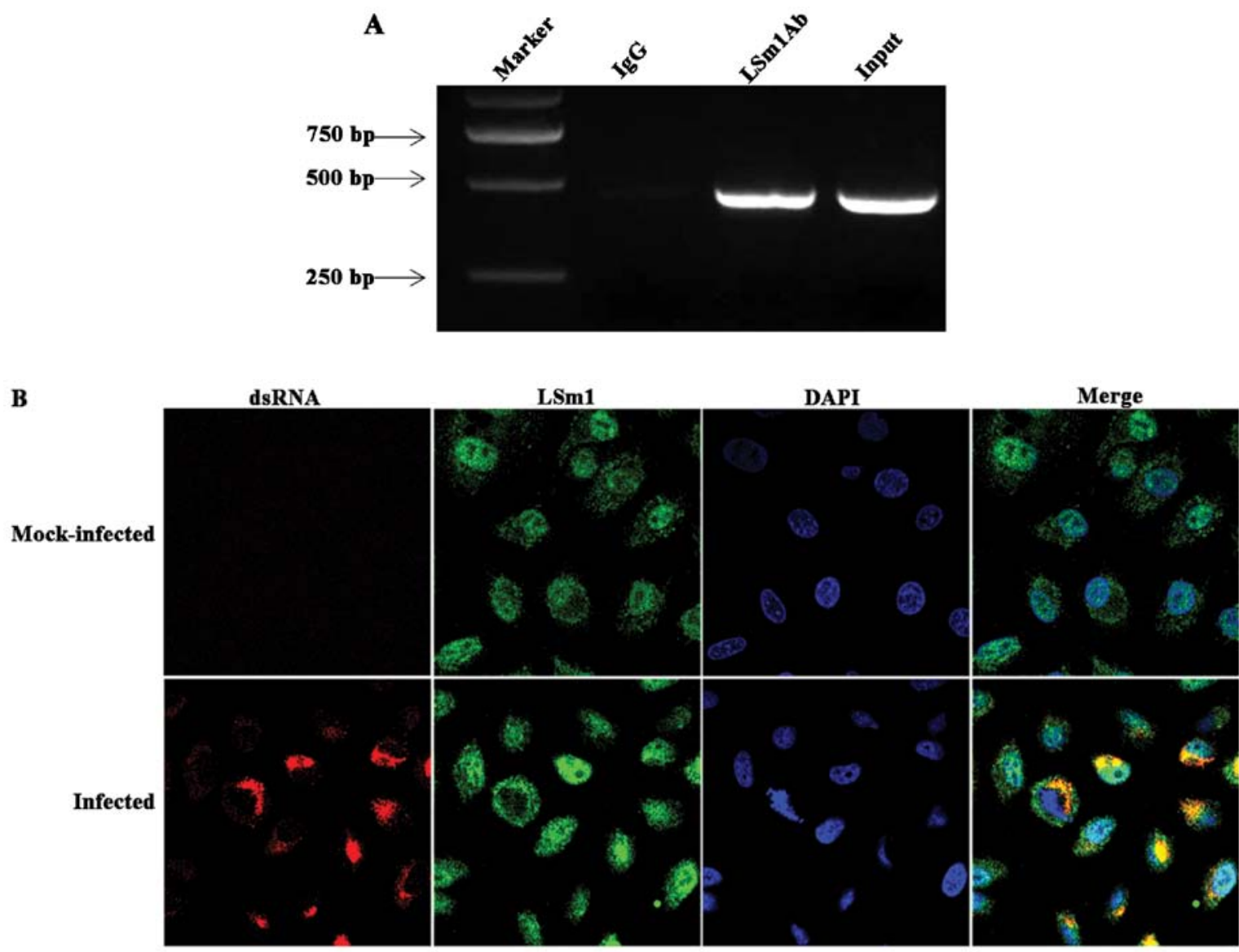

Figure 2. (A) Viral RNA was co-immunoprecipitated from Dengue virus (DENV)-infected A549 cell extracts using an anti-LSm1 antibody. Rabbit IgG was used as a negative control. RNA was extracted from the immunoprecipitated complex or from an aliquot of the input lysate and subjected to reverse transcription PCR (RT-PCR) using DENV 3' untranslated region (UTR) primers. (B) LSm1 intracellular localization in mock-infected and DENV-2-infected A549 cells. The cells were fixed at $24 \mathrm{~h}$ post-infection and were stained with the nuclear (blue) DAPI stain, with anti-dsRNA (red) and anti-LSm1 (green) antibodies. The merged panel shows an overlay of the DAPI, dsRNA and LSm1 signals. Colocalization of dsRNA and LSm1 is shown in yellow.

of the LSm1 protein and $74.33 \%$ of LSm1 mRNA when using the non-targeting siRNA siNC as a reference (Fig. 3A). At the time of maximal silencing, A549 cells were infected with DENV-2. After $36 \mathrm{~h}$, intracellular DENV RNAs were quantified by RT-qPCR, whereas cell supernatants were collected for the detection of infectious particles. Intracellular DENV RNA levels were significantly reduced by $59.43 \%$ compared to the siNC control while downregulating the LSm1 protein $71.71 \%$ (Fig. 3B). Moreover, DENV-2 production from siLsm1-transfected cells was also reduced by $36.24 \%$ compared to the siNC control (Fig. 3C). Decrease of viral RNA accumulation and particle production in LSm1 downregulation of cells indicated LSm1 is invovled in early stages of the viral life cycle, such as translation and replication.

Replication of DENV RNA in vivo is associated with P-body. P-bodies are distinct foci within the cytoplasm of the eukaryotic cell and play fundamental roles in mRNA translation, RNA silencing, and RNA degradation as well as viral infection processes (20-22). In the present study, we examined whether the positive regulating role of LSm1 in DENV RNA replication was associated with P-body. In the 5'-3'-deadenylation-dependent mRNA decay pathway, mRNA exit from translation and shortening of the poly(A) tail is followed by decapping via the Dcp1/Dcp2 decapping enzyme (23). This decapping process is not necessary for the HCV life cycle and Dcp protein is a commonly used marker to detect P-bodies $(16,24,25)$. We examined the subcellular localization of Dcp1B during DENV-2 infection. A549 cells were infected with DENV-2, and the cells were fixed, permeabilized and stained with anti-dsRNA and anti-Dcp1B antibody. The nuclei were stained with DAPI, and the coverslips were analyzed under a confocal laser scanning microscope. In the uninfected cells, we observed detectable Dcp1B exclusively. In the infected cells, Dcp1B colocalized with dsRNA signal (Fig. 4). This result showed that DENV RNA replicates reside within the P-bodies.

\section{Discussion}

Flavivirus 3' UTRs are known to have important regulatory significance for viral RNA replication and translation (26). Host factors binding 3' UTR-mediated RNA-protein interaction also play important roles in the flavivirus RNA replication. Several cell proteins interacting with DENV 3' UTR have been reported $(5-10,27)$. The LSm1-7 complexes have been identified to bind HCV 5' and 3' UTR, and are required for efficient HCV RNA translation and intracellular HCV RNA accumula- 
$\mathbf{A}$
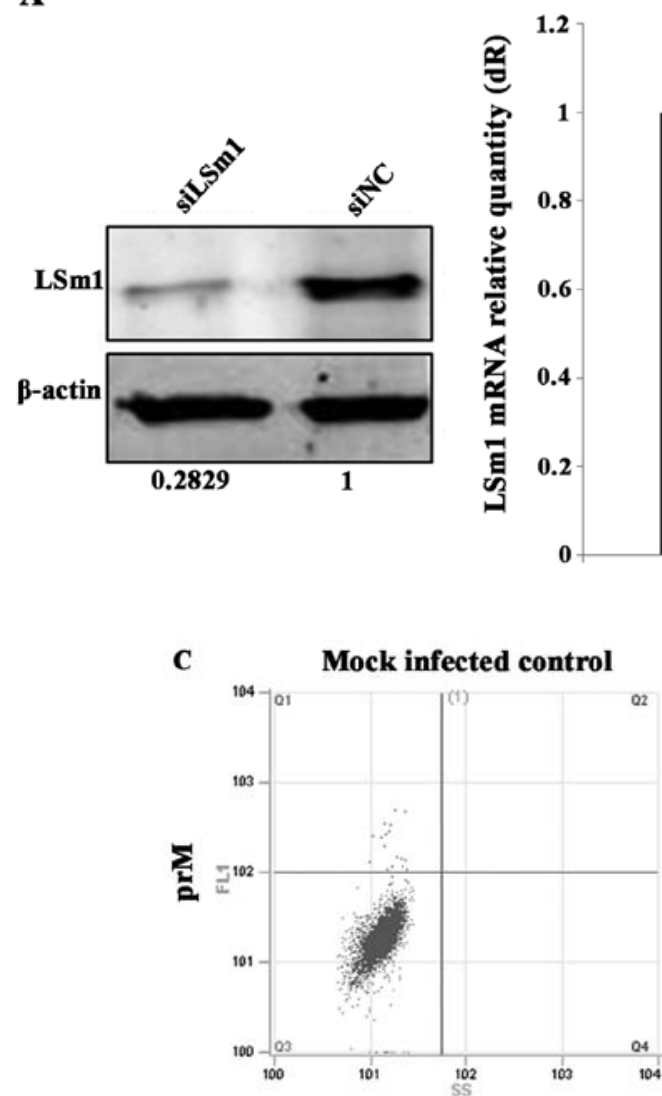

B

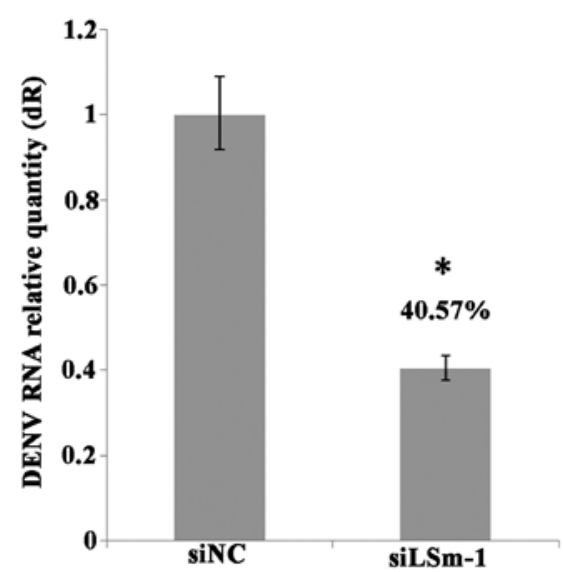

Figure 3. (A) LSm1 levels in control siNC cells and LSm1 knockdown cells. The siLSm1 contributed to a 71.71\% knockdown of LSm1 protein compared to the siNC control in A549 cells. (B) Viral RNA level in the Dengue virus (DENV)-2 infected LSm1 knockdown cells. LSm1 knockdown cells were infected with DENV. Total RNA was extracted, reverse transcribed, and used in relative reverse transcription quantitative PCR (RT-qPCR) reactions (target fragment was a partial NS5 fragment of the DENV genome and the internal control was the $\beta$-actin gene). DENV RNA level was significantly reduced by $59.43 \%$ in LSm1 knockdown cells. "P $<0.01$, which is considered statistically significant. (C) LSm1 knockdown reduces the production of infectious DENV. Supernatants from DENV-2-infected LSm1 knockdown cells were used to infect vero-E6 cells. At $36 \mathrm{~h}$ post-inoculation, the cells were collected and analyzed for viral infection by flow cytometry using antibodies against DENV prM. Supernatants from siNC- and siLSm1-treated cells showed a 24.36 and $15.34 \%$ infection rate, respectively.

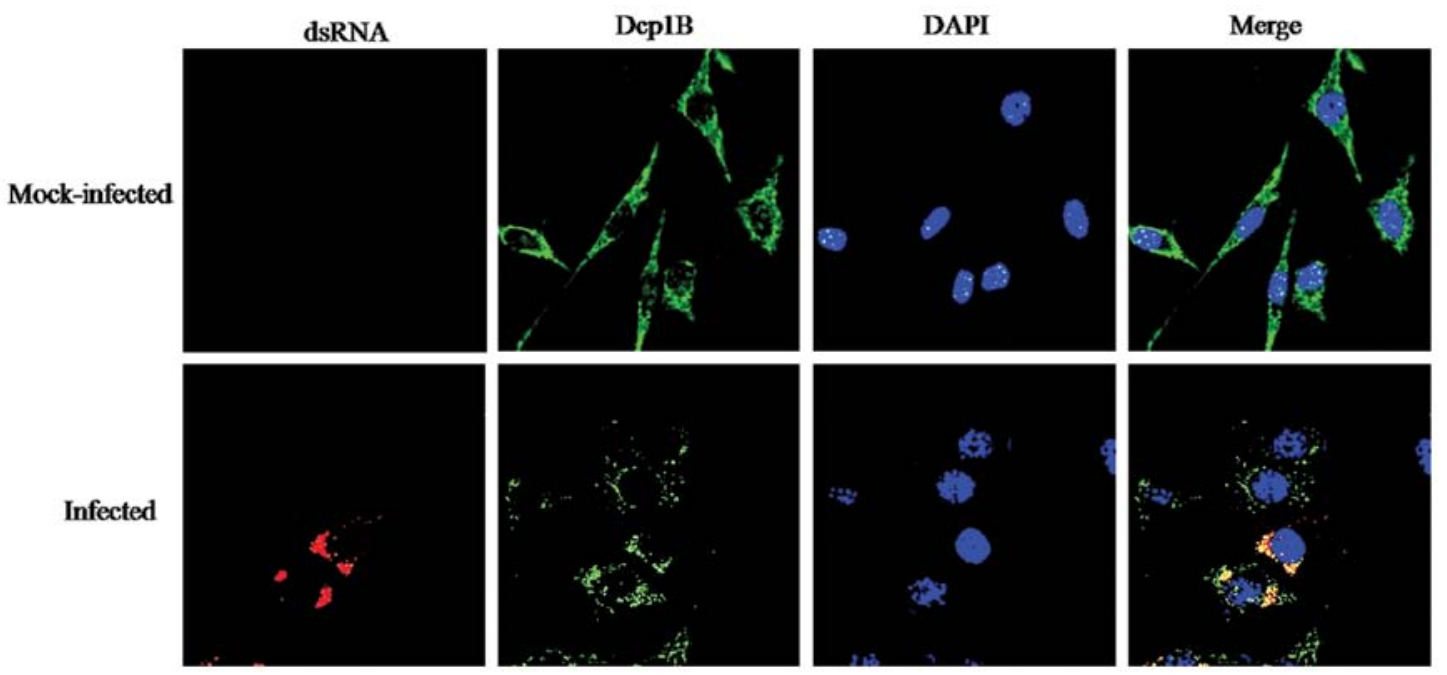

Figure 4. The P-body colocalizes with the Dengue virus (DENV) replication complex. Intracellular localization of Dcp1B, instead of LSm1, and dsRNA in mock-infected and DENV-2-infected A549 cells were observed under a confocal laser scanning microscopy, as described for Fig. 2B. The merged panel shows an overlay of the DAPI, dsRNA and Dcp1B signals. Colocalization of dsRNA and Dcp1B is shown in yellow foci.

tion $(15,16,25,28)$. By using two independent methodologies, we showed that LSm1 can bind 3' UTR of DENV-2 and as the positive regulator for viral RNA replication.
The Sm and Sm-like (LSm) proteins constitute a conserved family whose members function in multiple aspects of RNA metabolism. The LSm1-7 complex is localized in the cytoplasm 
and plays a key role in the regulation of the fate of cellular mRNAs from translation to degradation in the 5'-3'-deadenylation-dependent mRNA decay pathway $(11,12)$. In the present study, our data clearly demonstrate that LSm1-7 complexes are required for efficient DENV-2 propagation in human cells in culture. Downregulation of the LSm1-7 complexes significantly reduced DENV RNA replication and infectious viral particle release. It is known that the function of LSm1-7 complex as activator of the decapping of cellular mRNAs seems to involve their binding to short oligo(A) tracts at the 3 ' end of deadenylated cellular mRNAs (29). This interaction then inhibits trimming of the 3 ' end while simultaneously promoting decapping and subsequent degradation. However, the role of the LSm1-7 complexes on virus life cycles may be different as DENV 3 end does not contain the poly(A) tract. Accordingly, binding of the LSm1-7 complex to 3' UTR may facilitate rearrangement in the viral RNP structure required for the 5'-3' interaction. Long-range RNA-RNA interactions between the $5^{\prime}$ and $3^{\prime}$ ends, which circularize the DENV genome have been proposed to be required for RNA replication.

Notably, the LSm1-7 complex is a core component of P-body. P-bodies are highly dynamic cytoplasmic granules conserved among eukaryotes, which are sites at which non-translating mRNAs accumulate for different fates such as degradation, storage or returning to translation (20). It is known that several proteins within P-bodies or SGs can enhance or limit viral infection. Moreover, some viral RNAs and proteins, as well as host antiviral defense proteins, accumulate in P-bodies and/or SGs (30). Additionally, the LSm1-7 complex is thought to play a role in mRNP remodeling in P-body and this determines whether mRNAs within the P-body are to be translated, stored or degraded (31). By using confocal laser scanning microscopy, we observed the colocalization of the DENV dsRNA and Dcp1B, which indicates that the replication process occurs at the site of P-body. Together with the results that colocalization of the DENV dsRNA and LSm1-7 complex occurs in the perinuclear area, it is possible that the LSm1-7 complex binds to the DENV 3' UTR, and this RNP acts as a platform to recruit P-body components such as DDX6 and PatL1, and then form a P-body-like foci where replication occurs.

The interactions between the LSm1-7 complex and DENV RNA may facilitate rearrangements in the viral RNP structure and composition, recruiting proteins such as the LSm1-7 complex, DDX6 and PatL1 from the cellular mRNA repression/decay machinery and, instead of promoting decay, may promote viral RNA translation and subsequent transfer to replication. DDX6 has been shown to be involved in HCV and DENV RNA replication, and function as a positive regulator in the HCV and DENV viral cycle associated with the viral 3' UTR $(10,15,25,32)$. This view is consistent with another suggestion made for the regulation of DENV RNA. Ward et al (10) have shown that DDX6 interacts with DENV-2 RNA in vivo and binds to the DB2 and DB1 structures in the DENV-2 3' UTR. The interaction between DDX6 and the DB structures required the formation of a potential pseudoknot structure, suggesting that the complex tertiary structures in the DENV 3' UTR would be required for DDX6 function. Although the sites of DENV replication and translation have yet to be separated, it is possible that the LSm1-7 complex together with DDX6, key components of P-body, act to sort viral RNAs throughout the entire process, from translation and replication to the sites of viral packaging.

The results of the present study have demonstrated that the LSm1-7 complex, a cellular protein binding to the DENV RNA 3 ' UTR acts as a positive regulator of DENV replication. It is likely that the LSm1-7 complex functions directly or indirectly as an RBP rearranging the second structure of viral RNA to promote the formation of a complex suitable for replication.

\section{Acknowledgements}

This study was supported by the National Natural Science Foundation of China (NSFC 31370193).

\section{References}

1. Wiwanitkit V: Dengue fever: diagnosis and treatment. Expert Rev Anti Infect Ther 8: 841-845, 2010.

2. Rodenhuis-Zybert IA, Wilschut J and Smit JM: Dengue virus life cycle: viral and host factors modulating infectivity. Cell Mol Life Sci 67: 2773-2786, 2010.

3. Yocupicio-Monroy M, Padmanabhan R, Medina F and del Angel RM: Mosquito La protein binds to the 3' untranslated region of the positive and negative polarity dengue virus RNAs and relocates to the cytoplasm of infected cells. Virology 357: 29-40, 2007.

4. Yocupicio-Monroy RM, Medina F, Reyes-del Valle J and del Angel RM: Cellular proteins from human monocytes bind to dengue 4 virus minus-strand $3^{\prime}$ untranslated region RNA. J Virol 77: 3067-3076, 2003.

5. De Nova-Ocampo M, Villegas-Sepúlveda N and del Angel RM: Translation elongation factor-1alpha, $\mathrm{La}$, and PTB interact with the 3' untranslated region of dengue 4 virus RNA. Virology 295: 337-347, 2002.

6. Polacek C, Friebe P and Harris E: Poly(A)-binding protein binds to the non-polyadenylated 3 ' untranslated region of dengue virus and modulates translation efficiency. J Gen Virol 90: 687-692, 2009.

7. Gomila RC, Martin GW and Gehrke L: NF90 binds the dengue virus RNA 3' terminus and is a positive regulator of dengue virus replication. PLoS One 6: e16687, 2011.

8. Lei Y, Huang Y, Zhang H, Yu L, Zhang M and Dayton A: Functional interaction between cellular p100 and the dengue virus 3' UTR. J Gen Virol 92: 796-806, 2011.

9. Paranjape SM and Harris E: Y box-binding protein-1 binds to the dengue virus 3 -untranslated region and mediates antiviral effects. J Biol Chem 282: 30497-30508, 2007.

10. Ward AM, Bidet K, Yinglin A, Ler SG, Hogue K, Blackstock W, Gunaratne J and Garcia-Blanco MA: Quantitative mass spectrometry of DENV-2 RNA-interacting proteins reveals that the DEAD-box RNA helicase DDX6 binds the DB1 and DB2 3' UTR structures. RNA Biol 8: 1173-1186, 2011.

11. Beggs JD: Lsm proteins and RNA processing. Biochem Soc Trans 33: 433-438, 2005.

12. Khusial P, Plaag R and Zieve GW: LSm proteins form heptameric rings that bind to RNA via repeating motifs. Trends Biochem Sci 30: 522-528, 2005.

13. Diez J, Ishikawa M, Kaido M and Ahlquist P: Identification and characterization of a host protein required for efficient template selection in viral RNA replication. Proc Natl Acad Sci USA 97: 3913-3918, 2000.

14. Galao RP, Chari A, Alves-Rodrigues I, Lobão D, Mas A, Kambach C, Fischer U and Díez J: LSm1-7 complexes bind to specific sites in viral RNA genomes and regulate their translation and replication. RNA 16: 817-827, 2010.

15. Pérez-Vilaró G, Scheller N, Saludes V and Díez J: Hepatitis C virus infection alters P-body composition but is independent of P-body granules. J Virol 86: 8740-8749, 2012.

16. Scheller N, Mina LB, Galao RP, Chari A, Giménez-Barcons M, Noueiry A, Fischer U, Meyerhans A and Díez J: Translation and replication of hepatitis $C$ virus genomic RNA depends on ancient cellular proteins that control mRNA fates. Proc Natl Acad Sci USA 106: 13517-13522, 2009. 
17. Pang X, Zhang M and Dayton AI: Development of Dengue virus type 2 replicons capable of prolonged expression in host cells. BMC Microbiol 1: 18, 2001.

18. Polo S, Ketner G, Levis R and Falgout B: Infectious RNA transcripts from full-length dengue virus type 2 cDNA clones made in yeast. J Virol 71: 5366-5374, 1997.

19. Emara MM and Brinton MA: Interaction of TIA-1/TIAR with West Nile and dengue virus products in infected cells interferes with stress granule formation and processing body assembly. Proc Natl Acad Sci USA 104: 9041-9046, 2007.

20. Eulalio A, Behm-Ansmant I, Schweizer D and Izaurralde E: P-body formation is a consequence, not the cause, of RNAmediated gene silencing. Mol Cell Biol 27: 3970-3981, 2007.

21. Jakymiw A, Pauley KM, Li S, Ikeda K, Lian S, Eystathioy T, Satoh M, Fritzler MJ and Chan EK: The role of GW/P-bodies in RNA processing and silencing. J Cell Sci 120: 1317-1323, 2007.

22. Chan EK, Yao B and Fritzler MJ: Reflections on ten years of history of, and future prospects for, GW182 and GW/P body research. Adv Exp Med Biol 768: 261-270, 2013.

23. Coller J and Parker R: Eukaryotic mRNA decapping. Annu Rev Biochem 73: 861-890, 2004.

24. Yu SF, Lujan P, Jackson DL, Emerman M and Linial ML: The DEAD-box RNA helicase DDX6 is required for efficient encapsidation of a retroviral genome. PLoS Pathog 7: e1002303, 2011.

25. Ariumi Y, Kuroki M, Kushima Y, Osugi K, Hijikata M, Maki M Ikeda $\mathrm{M}$ and Kato $\mathrm{N}$ : Hepatitis $\mathrm{C}$ virus hijacks P-body and stress granule components around lipid droplets. J Virol 85: 6882-6892, 2011.
26. Men R, Bray M, Clark D, Chanock RM and Lai CJ: Dengue type 4 virus mutants containing deletions in the 3 ' noncoding region of the RNA genome: analysis of growth restriction in cell culture and altered viremia pattern and immunogenicity in rhesus monkeys. J Virol 70: 3930-3937, 1996.

27. Agis-Juárez RA, Galván I, Medina F, Daikoku T, Padmanabhan R, Ludert JE and del Angel RM: Polypyrimidine tract-binding protein is relocated to the cytoplasm and is required during dengue virus infection in Vero cells. J Gen Virol 90: 2893-2901, 2009.

28. Roberts AP, Doidge R, Tarr AW and Jopling CL. 2013. The $\mathrm{P}$ body protein $\mathrm{LSm} 1$ contributes to stimulation of hepatitis $\mathrm{C}$ virus translation, but not replication, by microRNA-122. Nucleic Acids Res 42: 1257-1269, 2014.

29. Chowdhury A, Mukhopadhyay J and Tharun S: The decapping activator Lsm1p-7p-Patlp complex has the intrinsic ability to distinguish between oligoadenylated and polyadenylated RNAs. RNA 13: 998-1016, 2007.

30. Beckham CJ and Parker R: P bodies, stress granules, and viral life cycles. Cell Host Microbe 3: 206-212, 2008.

31. Parker R and Sheth U: P bodies and the control of mRNA translation and degradation. Mol Cell 25: 635-646, 2007.

32. Huys A, Thibault PA and Wilson JA: Modulation of hepatitis C virus RNA accumulation and translation by DDX6 and miR-122 are mediated by separate mechanisms. PLoS One 8: e67437, 2013. 